\title{
Current Trends in Operative Treatment of Scaphotrapeziotrapezoid Osteoarthritis: A Survey among European Hand Surgeons
}

\author{
Merel J.-L. Berkhout, MD, MA ${ }^{1,2}$ Qiqi Yin, MD $^{3}$ Marco J. P. F. Ritt, MD, PhD ${ }^{2,3}$ \\ ${ }^{1}$ Department of Plastic, Reconstructive and Handsurgery, \\ Alrijne Hospital, Leiden, The Netherlands \\ 2 The Hand Clinic, Amsterdam, The Netherlands \\ ${ }^{3}$ Department of Plastic, Reconstructive and Hand Surgery, \\ Amsterdam UMC, Amsterdam, The Netherlands \\ Address for correspondence Merel J.-L. Berkhout, MD, MA, \\ Department of Plastic, Reconstructive and Hand Surgery, Alrijne \\ Hospital, P.O. Box 4220, 2350 CC Leiderdorp, The Netherlands \\ (e-mail: Berkhout@gmail.com).
}

J Wrist Surg 2020;9:94-99.

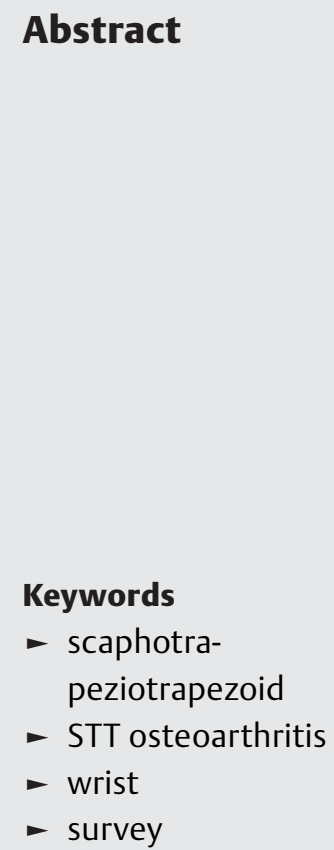

Abstract

The prevalence of isolated scaphotrapeziotrapezoid (STT) osteoarthritis (OA) increases with age and has been reported to range from 2 to $15 \%$ on wrist radiographs, ${ }^{1,2}$ and between 3 to $16 \%$ in cadaver studies., ${ }^{3,4}$ Symptomatic patients experience pain on the radial side of the wrist and the base of the thumb, limited range of motion (ROM), and decreased grip and pinch strength. Surgery might be indicated if conservative treatment fails. Surgical techniques include trapeziectomy with partial trapezoidal excision with or without ligament reconstruction and/or tendon interposition (LRTI), distal scaphoid excision with or without fibrous

Objective Based on the available evidence, the optimal surgical treatment for isolated scaphotrapeziotrapezoid oarthritis (STT OA) remains unclear. The purpose of this study is to explore the prevailing surgical practice for isolated STT OA among European hand surgeons. We hypothesized that a considerable variance exists in the current surgical practice among hand surgeons in Europe.

Methods An online survey was distributed to 20 participating member states of the Federation of European Societies for Surgery of the Hand (FESSH). Respondents were questioned on their country of practice, surgical experience, preferred surgical procedure, frequency of performing this procedure, factors in decision-making, and other performed surgical procedures.

Results Four hundred and sixty-five responses were received with an estimated response rate of $19 \%$. Trapeziectomy with partial trapezoidal excision was the surgical treatment of choice among the participating hand surgeons in Europe (38\%), followed by STT joint fusion (30\%), and distal scaphoid excision (14\%).

Conclusion This survey provides an insight into the surgical management of isolated STT OA among hand surgeons in Europe. A wide variety of preferred treatment techniques were found. Evidence concerning the optimal surgical treatment has not been established. Future prospective randomized studies comparing different techniques are warranted. interposition, STT joint fusion, implant arthroplasty, arthroscopic surgery, and surgical denervation. ${ }^{5-7}$

The optimal surgical treatment for isolated STT OA among hand surgeons in the United States showed a preference for trapeziectomy with LRTI. ${ }^{8}$

We hypothesized that considerable variance exists in the current surgical practice among hand surgeons in Europe. The purpose of this study is to explore the prevailing surgical practice for isolated STT OA among European hand surgeons. This includes determining the preferred surgical procedure, remains unclear. ${ }^{6}$ A survey by Wolf and Delaronde held received

October 27, 2018

accepted after revision

December 2, 2019

published online

February 12, 2020
Copyright $\odot 2020$ by Thieme Medical Publishers, Inc., 333 Seventh Avenue, New York, NY 10001, USA. Tel: +1(212) 760-0888.
DOI https://doi.org/ 10.1055/s-0039-3402796. ISSN 2163-3916. 
frequency of performing the technique, factors in decisionmaking, and other performed surgical procedures.

\section{Methods}

A survey consisting of demographic and treatment questions was constructed, based upon the current trends of surgical management of isolated STT OA. Demographic questions consisted of two single-answer multiple-choice questions. Treatment questions consisted of two single-answer multiplechoice questions, one multiple-choice question limited to two answers, and one multiple-answer multiple-choice question. Depending on the chosen answers, in-depth questions were asked to specify the technique used, potential complementary procedures and material used, or to elaborate on the factors that affect the decision-making. When appropriate, an "Other" option with write-in box was provided. A free-response item concluded the survey. The questions were compiled into an online survey using SurveyGizmo. A pilot group consisting of four experienced hand surgeons at the authors' institution was asked to evaluate question clarity and identify potential problems. Member states of the Federation of European Societies for Surgery of the Hand were approached for participation. An online link to the survey was electronically sent by the national delegates of the participating countries to their individual members. One reminder was distributed. Data collection took place between 11 October 2017 and 11 April 2018. The survey asked surgeons to identify practicerelated factors including country of practice and years of surgical experience. Then, the questions focused on the preferred surgical procedure, frequency of practice, and decisionmaking. Finally, participants were asked which other surgical procedures they perform for isolated STT OA.

\section{Data Analysis}

Data were analyzed with IBM SPSS Statistics for Windows Version 26 (IBM Corp., Armonk, NY). When appropriate, openended answers obtained from write-in boxes were categorized into original responses. Frequency distributions were created for all response variables and bivariate cross-tabulations were used to compare treatment variables with demographic variables. Comparison between the subgroups was done using the Pearson chi-squared test. Statistical significance was set at $p<0.05$.

\section{Results}

The survey was electronically delivered to roughly 2,500 members of 20 participating countries. We received 465 replies in total. Twenty-eight surgeons (6.0\%) did not treat STT OA surgically and there were 437 responses for the surgical treatment. The contribution of the participating countries and the experience of the surgeon are presented in - Tables 1 and 2. The estimated response rate was 19\%. Most responses came from Germany (24\%), the Netherlands (16\%), Switzerland (9.6\%), and France (9.4\%).

A summary of the respondents' preferred surgical procedure is listed in -Table 3 . Trapeziectomy with partial
Table 1 Survey demographics: country of practice

\begin{tabular}{|l|l|l|}
\hline $\begin{array}{l}\text { Country of } \\
\text { practice }\end{array}$ & $\begin{array}{l}\text { Response, } \\
\boldsymbol{n}=\mathbf{4 3 7}\end{array}$ & $\begin{array}{l}\text { Total } \\
\text { responses (\%) }\end{array}$ \\
\hline Austria & 25 & 5.7 \\
\hline Belgium & 26 & 5.9 \\
\hline Bulgaria & 3 & 0.7 \\
\hline Czech Republic & 13 & 3.0 \\
\hline Estonia & 5 & 1.1 \\
\hline Finland & 4 & 0.9 \\
\hline France & 41 & 9.4 \\
\hline Germany & 106 & 24.3 \\
\hline Greece & 20 & 4.6 \\
\hline Hungary & 17 & 3.9 \\
\hline Ireland & 1 & 0.2 \\
\hline Italy & 2 & 0.5 \\
\hline Latvia & 6 & 1.4 \\
\hline The Netherlands & 71 & 16.2 \\
\hline Norway & 8 & 1.8 \\
\hline Poland & 9 & 2.1 \\
\hline Portugal & 3 & 0.7 \\
\hline Sweden & 15 & 3.4 \\
\hline Switzerland & 42 & 9.6 \\
\hline Turkey & 20 & 4.6 \\
\hline & & \\
\hline & 20 & 2.6 \\
\hline
\end{tabular}

Table 2 Survey demographics: years of experience as hand surgeon

\begin{tabular}{|l|l|l|}
\hline $\begin{array}{l}\text { Years of experience } \\
\text { as hand surgeon }\end{array}$ & $\begin{array}{l}\text { Response, } \\
\boldsymbol{n = 4 3 7}\end{array}$ & $\begin{array}{l}\text { Total } \\
\text { responses (\%) }\end{array}$ \\
\hline$\leq 5$ & 74 & 16.9 \\
\hline $6-15$ & 175 & 40.0 \\
\hline $16-25$ & 122 & 27.9 \\
\hline$>25$ & 66 & 15.1 \\
\hline
\end{tabular}

trapezoidal excision is the treatment of choice among the participating hand surgeons in Europe (38\%), with the vast majority (70\%) performing additional LRTI. Variations in trapeziectomy with partial trapezoidectomy were performed, such as trapeziectomy alone and partial trapeziectomy with partial trapezoidectomy (-Table 3 , other). STT joint fusion was the second most preferred surgical procedure (30\%), followed by distal scaphoid excision (14\%). Half of the respondents favored fibrous interposition (capsule or tendon) after distal scaphoid excision (51\%), while the other half did not (49\%). Implant arthroplasty (8.5\%) and arthroscopic joint procedures $(4.1 \%)$ are the treatment of choice in a minority of respondents. Of the surgeons that chose implant arthroplasty (mainly the French surgeons), the Pyrocarbon implant is favored by the vast majority (83\%). Distal scaphoid excision is the most frequently performed arthroscopic procedure (50\%), followed by 
Table 3 Surgical management of STT OA: the preferred surgical procedure, other performed surgical procedures, and combined surgical practice

\begin{tabular}{|c|c|c|c|}
\hline Preferred surgical procedure & Preferred, $n=437,(\%)$ & Other, $n=425,(\%)$ & Overall, $n=437,(\%)$ \\
\hline \multicolumn{4}{|c|}{ Trapeziectomy with partial trapezoidal excision } \\
\hline LRTI: & $165(37.8)$ & $110(25.9)$ & $275(62.9)$ \\
\hline Yes & $115(70.1)$ & $79(73.1)$ & $194(71.3)$ \\
\hline No & $49(29.9)$ & 29 (26.9) & $78(28.7)$ \\
\hline STT joint fusion (arthrodesis) & $130(29.7)$ & $120(28.2)$ & $250(57.2)$ \\
\hline \multicolumn{4}{|c|}{ Distal scaphoid excision (open procedure) } \\
\hline Fibrous interposition: & $62(14.2)$ & $78(18.4)$ & $140(32.0)$ \\
\hline Yes & $31(50.8)$ & $35(44.9)$ & $66(47.5)$ \\
\hline No & $30(49.2)$ & $43(55.1)$ & $73(52.5)$ \\
\hline Implant arthroplasty & $37(8.5)$ & $34(8.0)$ & $71(16.2)$ \\
\hline Pyrocarbon & $30(83.3)$ & $25(73.5)$ & $55(78.6)$ \\
\hline Other & $6(16.7)$ & $9(26.5)$ & $15(21.4)$ \\
\hline Arthroscopic joint procedure & $18(4.1)$ & $33(7.8)$ & $51(11.7)$ \\
\hline Debridement & $3(16.7)$ & $11(33.3)$ & $14(27.5)$ \\
\hline Distal scaphoid excision & $9(50.0)$ & $12(36.4)$ & $21(41.2)$ \\
\hline $\begin{array}{l}\text { (Partial) trapeziectomy with } \\
\text { (partial) trapezoidal excision }\end{array}$ & $3(16.7)$ & $7(21.2)$ & $10(19.6)$ \\
\hline Other & $3(16.7)$ & $3(9.1)$ & $6(11.8)$ \\
\hline Surgical denervation & $6(1.4)$ & $75(17.6)$ & $81(18.5)$ \\
\hline Other & $19(4.3)$ & $15(3.5)$ & $34(7.8)$ \\
\hline None & & 78 (18.4) & \\
\hline
\end{tabular}

Abbreviations: LRTI, ligament reconstruction and/or tendon interposition; STT OA, scaphotrapeziotrapezoid osteoarthritis.

Notes: (1) Response counts in the columns "Other" and "Overall" do not add up to the total sum of participants, as respondents could opt for more than 1 "other" surgical procedure being part of their practice. (2) For 12 respondents only, the preferred surgical procedure was included in "Overall," due to partial completed questionnaires.

debridement and trapeziectomy with partial trapezoidal excision.

Of the 425 respondents that answered the question on whether they performed procedures other than their preferred procedure, 78 respondents did not perform any other procedure and 347 (79\%) performed other surgical procedures. When combining the preferred surgical procedure with the chosen other surgical procedures, trapeziectomy with partial trapezoidal excision, STT fusion, and distal scaphoid excision remained the preferred surgical practice for isolated STT OA. These procedures are chosen by 28,30 , and $14 \%$, respectively, of the respondents as the procedure of first choice and by 26,28 , and $18 \%$ as one of the other chosen procedures as part of their surgical management. Though one in five respondents (19\%) chose "surgical denervation" as a procedure they performed for isolated STT OA, only six respondents $(1.4 \%)$ chose this procedure as the treatment of choice. Most surgeons perform less than five distal scaphoid excisions $(74 \%)$ or STT fusions (78\%) ) each year. Trapeziectomy with partial trapezoidal excision was performed more frequently, with $41 \%$ of the respondents reported to perform this procedure 6 to 15 times yearly.

Of the countries with the most responses, trapeziectomy with partial trapezoidal excision appears to be the treatment of choice and was chosen particularly by hand surgeons practicing in Switzerland (57 vs. $36 \% ; p=0.006$ ) and the Netherlands ( 52 vs. $35 \% ; p=0.006$ ). This procedure is less popular in France (20 vs. $40 \% ; p=0.011$ ), Belgium (19 vs. $39 \% ; p=0.044$ ), and Turkey ( 10 vs. $39 \% ; p=0.009$ ). STT joint fusion remains popular in many countries, and is the preferred procedure among respondents in Turkey (80\%), Hungary (71\%), Sweden (47\%), Austria (44\%), and Czech Republic (54\%). In comparison to other countries, this procedure is less popular in France ( 7.3 vs. $32 \% ; p=0.001$ ), the Netherlands ( 5.6 vs. $34 \% ; p=0.0001$ ), and Switzerland (12 vs. $32 \% ; p=0.008)$. In France, implant arthroplasty is favored by over half of the surgeons (54\%), whereas distal scaphoid excision is the surgical treatment of choice among Belgian colleagues and chosen by $42 \%$ of the surgeons.

Implant arthroplasty is the only surgical procedure that is correlated to the surgeon's experience. None of the hand surgeons with less than 5 years of surgical experience chose this procedure as treatment of choice, in comparison to $10 \%$ of the hand surgeons with more than 5 years of surgical experience $(p=0.004)$.

Patient characteristics and familiarity with the procedure or training (-Table 4) were chosen by 49 and $45 \%$, 
Table 4 Decision-making: respondents choosing one of the given factors as one of the two most important factors in decision-making for the surgical procedure for STT OA

\begin{tabular}{|l|l|l|}
\hline Decision-making & Response & $\begin{array}{l}\text { Responses of } \\
\text { total participants } \\
\text { (\%), } \mathbf{n}=\mathbf{4 2 6}\end{array}$ \\
\hline $\begin{array}{l}\text { Familiarity with the } \\
\text { procedure/training }\end{array}$ & 192 & 45.1 \\
\hline $\begin{array}{l}\text { Hospital policy/ } \\
\text { department protocol }\end{array}$ & 18 & 4.2 \\
\hline Patient characteristics & 208 & 48.8 \\
\hline $\begin{array}{l}\text { Procedure related } \\
\text { benefits }\end{array}$ & 151 & 35.4 \\
\hline Preoperative findings & 159 & 37.3 \\
\hline $\begin{array}{l}\text { DISI stance on } \\
\text { lateral X-ray }\end{array}$ & 30 & 18.9 \\
\hline Severity of OA & 120 & 75.5 \\
\hline $\begin{array}{l}\text { Excessive dorsal/volar } \\
\text { translation of the wrist }\end{array}$ & 3 & 1.9 \\
\hline Other & 6 & 3.8 \\
\hline Other & 3 & 0.7 \\
\hline
\end{tabular}

Abbreviations: DISI, dorsal intercalated segment instability; STT OA, scaphotrapeziotrapezoid osteoarthritis.

Note: Response counts do not add up to the total sum of participants $(n=426)$, as respondents could opt for 1 or 2 factors.

respectively, of the respondents as the two most important factors influencing the decision-making when considering surgery for STT OA. Of the respondents that chose preoperative findings, the majority (76\%) chose severity of OA as the most important with $19 \%$ of the respondents choosing a dorsal intercalated segment instability (DISI) on lateral radiograph as the most important preoperative factor in their decision-making.

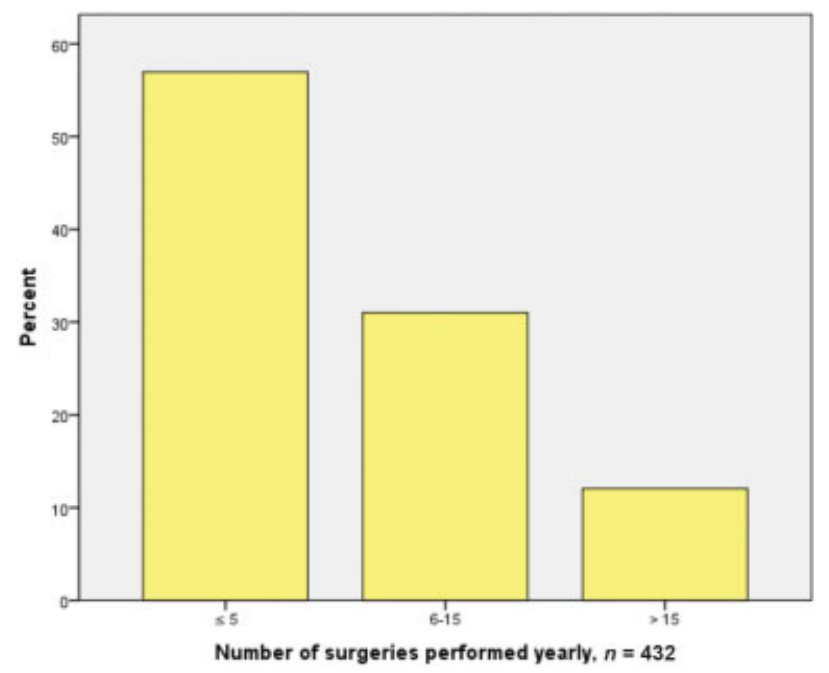

Fig. 1 Surgeries performed in the last year.

\section{Discussion}

The literature focuses on six different techniques for the surgical treatment of isolated STT OA. ${ }^{5-7}$ Published studies are scarce and encompass retrospective data with small sample sizes. Deans et al in their systematic review found only 21 eligible papers, all level 4 evidence. ${ }^{6}$ In this study, trapeziectomy with partial trapezoidal excision is the preferred procedure among the participating hand surgeons in Europe. In the study by Andrachuk and Yang, 10 patients (12 wrists) with isolated STT OA underwent trapeziectomy and proximal trapezoid excision with LRTI. After an average follow-up of 18 months, pain scores decreased and ROM increased uniformly. ${ }^{9}$ It has been hypothesized that additional partial trapezoid excision may not be necessary, as one study on trapeziectomy and LRTI showed good objective and subjective results at a mean follow-up of 54 months for 14 consecutive patients (15 wrists) with isolated STT OA. ${ }^{10}$ Prospective randomized studies comparing trapeziectomy with and without partial trapezoid excision are necessary to evaluate this hypothesis.

STT fusion is a popular procedure, with more than half of the respondents reporting this procedure being part of their surgical practice. STT fusion is a technically demanding procedure. Complications include radiocarpal OA, radial styloid impingement, trapeziometacarpal OA, and nonunion, with nonunion rates varying from 4 to $31 \% .^{11-13}$ It also causes a decrease in postoperative ROM, with reported ROM varying from $75 \%$ of the opposite unaffected side after STT fusion to a flexion- extension arc of $81 \%$ and a radioulnar arc of $68 \%$ of the preoperative range after a mean follow-up of 4 years. ${ }^{12-14}$ However, good pain relief and grip strength could be achieved with STT fusion.

Distal scaphoid excision is the third most popular procedure among hand surgeons in Europe. Studies show that distal scaphoid excision results in good pain relief. ${ }^{15,16}$ Reported complications of the procedure are not common, but include radial nerve irritation, injuries to the radial artery, and rupture of the flexor digitorum superficialis and flexor digitorum profundus to the index finger. ${ }^{17,18}$ The occurrence of a static or dynamic DISI pattern of carpal instability as a result of distal scaphoid resection has been reported in several studies. ${ }^{15,16,19}$ Tendon interposition did not to affect the degree of DISI deformity. ${ }^{16}$ Patients without fibrous interposition after distal scaphoid excision showed significantly greater wrist flexionextension motion than patients with soft-tissue interposition. ${ }^{15}$ No correlation was found between DISI stance, pain scores, and functional outcomes. ${ }^{15,16}$ In the presence of clinical or subclinical midcarpal instability, alternative procedures have been suggested. ${ }^{6}$ A Pyrocarbon implant to fill the gap may help to prevent carpal instability. ${ }^{19}$

In this survey, implant arthroplasty was mainly performed in France. Compared with distal scaphoid excision, the main advantage of this procedure is that it retains scaphotrapezial mobility without destabilizing the carpal bones. Published studies reported functional improvement and decrease in pain scores postoperatively, but changes in carpal alignment on X-ray were not reported. ${ }^{20-23}$ Amplitudes of ROM were 
maintained except for wrist extension and radial deviation, which were diminished by less than 15 and 10 , degrees respectively. ${ }^{23}$ Grip and pinch strength remained largely preserved; one study reported this to be 85 and $82 \%$ respectively compared with the nonoperated side. ${ }^{21}$ Implant dislocation may be a complication of implant arthroplasty. However, in cases of failure, other revision procedures may be considered.

In our survey, most surgeons, when performing arthroscopic surgery for STT OA, opted for distal scaphoid excision. Studies that reported this technique show improvement in pain, grip, and pinch strength and mobility postoperatively. ${ }^{22,24,25}$ In a recent prospective study of 11 patients who underwent arthroscopic resection and 13 patients who underwent additional Pyrocarbon implant interposition the Disabilities of the Arm, Shoulder and Hand (DASH) and visual analog scale (VAS) scores, grip and pinch strength, and X-rays for DISI stance were comparable 24 months postoperatively. ${ }^{22}$ These findings are in contrast to another study revealing a significant reduction in pinch strength among patients with a Pyrocarbon implant, compared with patients without Pyrocarbon implant. ${ }^{24}$ Other procedures performed arthroscopically are debridement and trapeziectomy with partial trapezoidal excision. Ashwood et al compared ROM, grip strength, VAS score, and satisfaction before and after arthroscopic debridement. In their study, patients achieved good symptomatic relief at a mean followup of 36 months. $^{26}$

Surgical denervation for isolated STT OA is performed by $19 \%$ of all respondents. Braga-Silva et al performed a retrospective review of 49 wrist denervations for degenerative $O A$ of the wrist caused by scaphoid nonunion advanced collapse, Kienbö ck disease, and primary degenerative OA. At 36 months after surgery, the average improvement in pain scores was $79 \pm 4 \%$ for all groups. Grip strength on the treated side improved from 43 to $69 \%$ of the opposite side and measurements of ROM showed improvement in all axes of movement. Another retrospective study ${ }^{7}$ included 71 complete denervations of the wrist joint in 70 patients with different treatment indications including scaphoid nonunions, distal radius fracture, Kienbö ck disease, primary degenerative arthritis, and scapholunate dissociation. After an average follow-up period of 9.6 years, subjective improvement using the DASH questionnaire was reported in two-thirds, and pain relief in half of the patients. ${ }^{5,7}$ Although the surgical indications in these studies do not include isolated STT OA, wrist denervation may be considered as a simple and fast pain-relieving procedure that does not affect the ROM.

The clinical practice of hand surgeons in Europe as obtained from our survey largely resembles the practice in the United States. A survey held in 2012 among hand surgeons in the United States concerning trapeziometacarpal OA included one question about the treatment of STT OA in a 62-year-old male computer specialist with failed conservative treatment. ${ }^{26}$ Half of the 1,156 participating respondents (50\%) would perform trapeziectomy and LRTI, followed by STT fusion (28\% ) and distal scaphoid excision (12\% ). Only $9 \%$ of the respondents were willing to perform other procedures. Compared with the United States, the respondents from Europe perform less trapeziectomy with partial trape- zoidectomy and LRTI. Instead, alternative techniques such as implant arthroplasty and arthroscopy are more often chosen as the preferred surgical procedure.

Among all respondents, patient characteristics are considered to be one of the two most important factors for decision-making, followed by familiarity with the procedure or training. Also, hospital policy or department protocol is mentioned by several surgeons as influencing their decision. The latter two choices reflect that expert opinion is still pivotal in decision-making.

A limitation of our study is the estimated response rate of $19 \%$. Response rates among participating countries differed and several member states did not participate. A general limitation of questionnaires is that participants often choose the answer representing what they believe they "should do" instead of what they "actually do." We attempted to minimize this confounder by anonymizing all responses.

We are not aware of previous European-wide studies that provide insight to the controversial surgical management of isolated STT OA. We attempted to obtain a genuine representation of actual practice by asking questions about both the preferred surgical procedure and other performed procedures. We also attempted to clarify the reasoning behind the surgeons' decisions. Surgeons had the option to deviate from the fixed multiple-choice options in open-ended writein boxes, and share their personal experience.

For now, based on current literature, there is no evidence to support one procedure over another and expert opinion remains crucial in decision-making. The authors agree with the participating surgeons: patient characteristics and preoperative findings are very important factors in choosing the best option for our patients. In our opinion, trapeziectomy with partial trapezoidectomy is a good option in cases of combined STT and trapeziometacarpal OA. For patients with isolated STT OA and preexisting carpal instability and for patients involved in high demand manual labor, a distal scaphoidectomy should be avoided. STT fusion, in spite of the high complication rate, may be a better option for this group as it provides a more stable construct. In contrast, for low demand patients without carpal instability, distal scaphoidectomy either open or arthroscopically does seem a suitable treatment option. Implant arthroplasty, the most expensive option, is useful for both stable and unstable wrists, but the long-term results and survivability of the implants are as yet unclear. Denervation may be a good and simple option; however, there is no literature to support its use for STT OA.

Future long-term studies, preferably comparing different techniques, are necessary to ultimately find the best surgical procedure for isolated STT OA.

Note

The work was performed at the Department of Plastic, Reconstructive and Hand Surgery, Amsterdam UMC, the Netherlands.

Funding

None. 


\section{Conflict of Interest}

None declared.

\section{References}

1 Armstrong AL, Hunter JB, Davis TRC. The prevalence of degenerative arthritis of the base of the thumb in post-menopausal women. J Hand Surg [Br] 1994;19(03):340-341

2 Scordino LE, Bernstein J, Nakashian M, et al. Radiographic prevalence of scaphotrapeziotrapezoid osteoarthrosis. J Hand Surg Am 2014;39(09):1677-1682

3 Brown GD III, Roh MS, Strauch RJ, Rosenwasser MP, Ateshian GA, Mow VC. Radiography and visual pathology of the osteoarthritic scaphotrapezio-trapezoidal joint, and its relationship to trapeziometacarpal osteoarthritis. J Hand Surg Am 2003;28(05):739-743

4 North ER, Eaton RG. Degenerative joint disease of the trapezium: a comparative radiographic and anatomic study. J Hand Surg Am 1983;8(02):160-166

5 Braga-Silva J, Román JA, Padoin AV. Wrist denervation for painful conditions of the wrist. J Hand Surg Am 2011;36(06): 961-966

6 Deans VM, Naqui Z, Muir LT. Scaphotrapeziotrapezoidal joint osteoarthritis: a systematic review of surgical treatment. J Hand Surg Asian Pac Vol 2017;22(01):1-9

7 Schweizer A, von Kä nel O, Kammer E, Meuli-Simmen C. Longterm follow-up evaluation of denervation of the wrist. J Hand Surg Am 2006;31(04):559-564

8 Wolf JM, Delaronde S. Current trends in nonoperative and operative treatment of trapeziometacarpal osteoarthritis: a survey of US hand surgeons. J Hand Surg Am 2012;37(01):77-82

9 Andrachuk J, Yang SS. Modified total trapezial and partial trapezoidal excision and ligament reconstruction tendon interposition reduces symptoms in isolated scaphotrapezial-trapezoid arthritis of the wrist. J Hand Surg Eur Vol 2012;37(07): 637-641

10 Langenhan R, Hohendorff B, Probst A. Trapeziectomy and ligament reconstruction tendon interposition for isolated scaphotrapeziotrapezoid osteoarthritis of the wrist. J Hand Surg Eur Vol 2014;39(08):833-837

11 Fortin PT, Louis DS. Long-term follow-up of scaphoid-trapeziumtrapezoid arthrodesis. J Hand Surg Am 1993;18(04):675-681

12 Goubier JN, Bauer B, Alnot JY, Teboul F. Traitement de l'arthrose scaphotrapé zotrapé zoidienne isolé e par arthrodè se scaphotrapé zotrapé zoidienne: une sé rie de 13 cas [scapho-trapezio-trapezoidal arthrodesis for scapho-trapezio-trapezoidal osteoarthritis]. Chir Main 2006;25(05):179-184
13 Watson HK, Weinzweig J, Guidera PM, Zeppieri J, Ashmead D. One thousand intercarpal arthrodeses. J Hand Surg [Br] 1999;24(03): 307-315

14 Meier R, Prommersberger KJ, Krimmer H. [Scapho-trapezio-trapezoid arthrodesis (triscaphe arthrodesis)]. Handchir Mikrochir Plast Chir 2003;35(05):323-327

15 Garcia-Elias M, Lluch AL, Farreres A, Castillo F, Saffar P. Resection of the distal scaphoid for scaphotrapeziotrapezoid osteoarthritis. J Hand Surg [Br] 1999;24(04):448-452

16 Berkhout MJL, Bachour Y, Wessing D, Ritt MJPF. Distal pole resection of the scaphoid for the treatment of scaphotrapeziotrapezoid osteoarthritis. Hand (N Y) 2019;14(02):230-235

17 Deren ME, Mitchell CH, Weiss AC. Flexor tendon ruptures after distal scaphoid excision for scaphotrapeziotrapezoid osteoarthritis. Hand (N Y) 2017;12(05):NP152-NP156

18 Wessels KD. Arthroplastik bei Arthrose im Triskaphoidgelenk nach Koob [Arthroplasty of osteoarthritic triscaphe (distal scaphoid) joint]. Oper Orthop Traumatol 2004;16(01):48-58

19 Marcuzzi A, Ozben H, Russomando A. Treatment of scaphotrapezial trapezoidal osteoarthritis with resection of the distal pole of the scaphoid. Acta Orthop Traumatol Turc 2014;48(04):431-436

20 Gauthier E, Truffandier MV, Gaisne E, Bellemè re P. Treatment of scaphotrapeziotrapezoid osteoarthritis with the Pyrocardan ${ }^{\circledR}$ implant: Results with a minimum follow-up of 2 years. Hand Surg Rehabil 2017;36(02):113-121

21 Low AK, Edmunds IA. Isolated scaphotrapeziotrapezoid osteoarthritis: preliminary results of treatment using a pyrocarbon implant. Hand Surg 2007;12(02):73-77

22 Pegoli L, Pozzi A, Pivato G, Luchetti R. Arthroscopic resection of distal pole of the scaphoid for scaphotrapeziotrapezoid joint arthritis: comparison between simple resection and implant interposition. J Wrist Surg 2016;5(03):227-232

23 Pequignot JP, D' asnieres de Veigy L, Allieu Y. Traitement de l' arthrose S.T.T. par un Implant en pyrocarbone. Premiers ré sultats [arthroplasty for scaphotrapezoidal arthrosis using a pyrolitic carbon implant, preliminary results]. Chir Main 2005;24(3-4):148-152

24 Mathoulin C, Darin F. Arthroscopic treatment of scaphotrapeziotrapezoid osteoarthritis. Hand Clin 2011;27(03):319-322

25 Normand J, Desmoineaux P, Boisrenoult P, Beaufils P. Ré section arthroscopique du pôle distal du scaphoï de dans l'arthrose scapho-trapé zo-trapé zoidienne [the anthroscopic distal pole resection of the scaphoid: clinical results in STT osteoarthritis]. Chir Main 2012;31(01):13-17

26 Ashwood N, Bain GI, Fogg Q. Results of arthroscopic debridement for isolated scaphotrapeziotrapezoid arthritis. J Hand Surg Am 2003;28(05):729-732 